\title{
Three-dimensional simulations of the interaction between Type la supernova ejecta and their main sequence companions
}

\author{
Z. W. Liu ${ }^{1,2,3,4}$, R. Pakmor ${ }^{5,4}$, F. K. Röpke ${ }^{6,4}$, P. Edelmann ${ }^{4}$, B. Wang ${ }^{1,2}$, M. Kromer ${ }^{4}$, \\ W. Hillebrandt ${ }^{4}$, and Z. W. Han ${ }^{1,2}$
}

\author{
${ }^{1}$ National Astronomical Observatories/Yunnan Observatory, Chinese Academy of Sciences, 650011 Kunming, PR China \\ 2 Key Laboratory for the Structure and Evolution of Celestial Objects, Chinese Academy of Sciences, 650011 Kunming, PR China \\ ${ }^{3}$ University of Chinese Academy of Sciences, 100049 Beijing, PR China \\ ${ }^{4}$ Max-Planck-Institut für Astrophysik, Karl-Schwarzschild-Str. 1, 85741 Garching, Germany \\ e-mail: zwliu@mpa-garching.mpg.de \\ 5 Heidelberger Institut für Theoretische Studien, Schloss-Wolfsbrunnenweg 35, 69118 Heidelberg, Germany \\ ${ }^{6}$ Institut für Theoretische Physik und Astrophysik, Universität Würzburg, Emil-Fischer-Str. 31, 97074 Würzburg, Germany
}

Received 5 April 2012 / Accepted 18 September 2012

\section{ABSTRACT}

\begin{abstract}
Context. The identity of the progenitor systems of Type Ia supernovae (SNe Ia) is still uncertain. In the single-degenerate scenario, the interaction between the supernova blast wave and the outer layers of a main sequence companion star strips off hydrogen-rich material which is then mixed into the ejecta. Strong contamination of the supernova ejecta with stripped material could lead to a conflict with observations of SNe Ia. This constrains the single-degenerate progenitor model.

Aims. In this work, our previous simulations based on simplified progenitor donor stars have been updated by adopting more realistic progenitor-system models that result from fully detailed, state-of-the-art binary evolution calculations.

Methods. We use Eggleton's stellar evolution code including the optically thick accretion wind model and taking into account the possibility of the effects of accretion disk instabilities to obtain realistic models of companion stars for different progenitor systems. The impact of the supernova blast wave on these companion stars is followed in three-dimensional hydrodynamic simulations employing the smoothed particle hydrodynamics code GADGET3.

Results. For a suite of main sequence companions, we find that the mass of the material stripped from the companions range from $0.11 M_{\odot}$ to $0.18 M_{\odot}$. The kick velocity delivered by the impact is between $51 \mathrm{~km} \mathrm{~s}^{-1}$ and $105 \mathrm{~km} \mathrm{~s}^{-1}$. We find that the stripped mass and kick velocity depend on the ratio of the orbital separation to the radius of a companion, $a_{\mathrm{f}} / R$. They can be fitted in good approximation by a power law for a given companion model. However, we do not find a single power law relation holding for different companion models. This implies that the structure of the companion star is also important for the amount of stripped material.

Conclusions. With more realistic companion star models than those employed in previous studies, our simulations show that the hydrogen masses stripped from companions are inconsistent with the best observational limits $\left(\leqslant 0.01 M_{\odot}\right)$ derived from SN Ia nebular spectra. However, a rigorous forward modeling from the results of impact simulations with radiation transfer is required to reliably predict observable signatures of the stripped hydrogen and to conclusively assess the viability of the considered SN Ia progenitor scenario.
\end{abstract}

Key words. supernovae: general - hydrodynamics - binaries: close

\section{Introduction}

Type Ia supernovae (SNe Ia) play a fundamental role in astrophysics. They are one of the most powerful tools in cosmology due to their extreme luminosities and high homogeneity. Based on an empirical relation between the light curve shape and the peak luminosity (Phillips 1993; Phillips et al. 1999), they can be used as accurate cosmic distance indicators. This led to the discovery of the accelerating expansion of the Universe (Riess et al. 1998; Perlmutter et al. 1999; Leibundgut 2008). However, despite recent progress on both, the theoretical and observational side, the nature of SN Ia progenitors and the physics of the explosion mechanisms are still unclear (Hillebrandt \& Niemeyer 2000).

It is widely accepted that $\mathrm{SNe}$ Ia arise from a mass-accreting white dwarf (CO WD) undergoing a thermonuclear explosion (for a review see Hoyle \& Fowler 1960; Finzi \& Wolf 1967; Nomoto 1982). At present, the most general classification of progenitor scenarios are single-degenerate and doubledegenerate models. In the single degenerate (SD) scenario, a white dwarf (WD) accretes hydrogen-rich material from its non-degenerate companion, where the companion star could be a main-sequence (MS) star (WD + MS channel), a slightly evolved subgiant star or a red giant (RG) star. When the mass of the WD approaches the Chandrasekhar mass limit, it explodes as a SN Ia (e.g. Whelan \& Iben 1973; Hachisu et al. 1996; Han \& Podsiadlowski 2004; Wang et al. 2010). In the double degenerate (DD) scenario, two CO WDs spiral in and merge due to gravitational wave radiation, resulting in a single object with a mass above the Chandrasekhar limit, which then may explode as SN Ia (Iben \& Tutukov 1984; Webbink 1984).

However, neither the SD nor the DD models can yet be clearly favored from observation or theory (Maoz \& Mannucci 2012). In the SD case, only a fairly narrow range in the accretion rate above $10^{-7} M_{\odot} \mathrm{yr}^{-1}$ is allowed to attain stable hydrogen burning on the surface of the WD, avoiding a nova explosion. 
This makes it difficult to explain the observed nearby SN Ia rate (Mannucci 2005; Maoz \& Mannucci 2012). Recent observations have identified some DD binaries (Nelemans et al. 2005; Geier et al. 2007). However, only few DD systems have been found whose orbital period is short enough to merge in a Hubble time (Geier et al. 2010; Rodríguez-Gil et al. 2010). In none of them, the combined mass exceeds the Chandrasekhar limit. In addition, the DD channel has been suggested to lead to an accretioninduced collapse rather than an SN Ia (Nomoto \& Iben 1985; Saio \& Nomoto 1998). Recently, however, Pakmor et al. (2010, 2011) found that violent mergers of equal-mass white dwarfs with masses $\sim 0.9 M_{\odot}$ can directly trigger thermonuclear explosions which resemble sub-luminous 1991 bg-like SNe Ia. The violent merger of two CO WDs with masses of $0.9 M_{\odot}$ and $1.1 M_{\odot}$ can lead to events that reproduce observational characteristics of normal SNe Ia (Pakmor et al. 2012b).

With detailed binary population synthesis (BPS) calculations, some research groups investigated the delay-time distributions (DTD) and birthrates of SNe Ia for different formation channels. Theoretical predictions are compared with observations to constrain the progenitor systems of SNe Ia and their forming scenarios (Ruiter et al. 2009; Mannucci 2009; Wang et al. 2009; Wang \& Han 2010; Maoz \& Badenes 2010; Maoz et al. 2010). However, there is no evidence that the SD scenario alone explains the shape of the observed delay-time distribution, while this may be possible for the DD scenario or a combination of both channels (Mennekens et al. 2010).

The obvious difference between the SD and DD scenario is that the companion stars in the SD channel will survive and may affect the observational display of the explosion (Marietta et al. 2000; Pakmor et al. 2008). In contrast, no stellar remnant exists in the case of the merger of two WDs in the DD channel. It is a promising approach to constrain the nature of SN Ia progenitors by directly searching for the surviving companion stars in remnants of SNe Ia. A prominent example is Tycho Brahe's supernova (SN 1572) for which Ruiz-Lapuente et al. (2004) presented a survey of the central region of its remnant, around the position of the explosion. They identified a subgiant star (Tycho G), similar to the Sun in surface temperature but with very low surface gravity, which moves at more than three times the mean velocity of the stars in the neighborhood and suggested that this might be the surviving companion of SN 1572 . However, the study of Kerzendorf et al. (2009) casts some doubts on this identification. Recently, Schaefer \& Pagnotta (2012) analyzed the center of the Large Magellanic Cloud SN Ia remnant SNR 0509-67.5 which does not contain any stars down to the observational limit. Thus, all possible companion star types except white dwarfs can be excluded for this object.

In the SD scenario, hydrogen-rich or helium-rich circumstellar material (CSM) is expected to exist around SNe Ia as the result of mass transfer from the companion, as well as WD winds (Nomoto 1982; Hachisu et al. 1999b). Therefore, another indirect way of identifying SN Ia progenitor systems is to search for the material transferred to the accreting white dwarf in the CSM (Patat et al. 2007). Following this approach Patat et al. (2007) reported detection of such CSM. They suggested that the SD system consisting of a WD and RG is the favored progenitor for SN 2006X (Patat et al. 2007). Moreover, Sternberg et al. (2011) showed that the velocity structure of absorbing material along the line of sight to $35 \mathrm{SNe}$ Ia tends to be blueshifted. Thus, they concluded that many SNe Ia in nearby spiral galaxies may originate in SD systems (Sternberg et al. 2011). Note, however, that abundant CSM is in conflict with the missing radio signal for other events, e.g. SN 2011 fe (Chomiuk et al. 2012; Horesh et al. 2012).

SNe Ia are characterized by the lack of hydrogen in their spectra. This is explained naturally in the DD progenitor scenario. However, in the SD scenario, the companion star is typically hydrogen-rich. Thus, the impact of a SN Ia explosion will strip off hydrogen-rich material from the companion and mix it into the ejecta, which may be in conflict with observations if the amount of hydrogen is large enough to be observable. Thus, we may be able to identify the progenitors of $\mathrm{SNe}$ Ia based on the exact amount of hydrogen stripped from the surface of the companion star. So far, there is no direct detection of the stripped hydrogen. Based on high-resolution spectroscopy of the two extragalactic SNe Ia SN 2005am and SN 2005cf, Leonard (2007) estimated an upper limit of $0.01 M_{\odot}$ applying the model described by Mattila et al. (2005).

Marietta et al. (2000; hereafter M00) explored the impact of SN Ia ejecta on a variety of binary companions (MS, subgiants, RGs) in the SD formation channel with two dimensional Eulerian hydrodynamics simulations. They found that the supernova explosion can strip $0.15 M_{\odot}$ to $0.17 M_{\odot}$ of material from the surface of MS and subgiant companions, while RGs lose almost their entire envelope in the impact. However, they ignored the effect of the mass transfer phase on the structure of the companion star when they constructed their initial model. To investigate how the mass transfer changes the mass stripping by supernova explosions, Meng et al. (2007) used an analytical method to approximate the mass loss in the impact. They found a lower limit of $0.035 M_{\odot}$ for the stripped mass, but their analytic method was based on an oversimplified description of the interaction physics.

An updated study has been presented by Pakmor et al. (2008, hereafter PRWH08) based on three-dimensional (3D) smoothed particle hydrodynamics (SPH) simulations. By mimicking the models of Ivanova \& Taam (2004) in their setups, they found stripped masses in the range from $0.01 M_{\odot}$ to $0.06 M_{\odot}$, which is very close to the observational limits of $\sim 0.01 M_{\odot}$. Therefore, they concluded that the SD scenario remains a valid possibility for SN Ia progenitors. Although mass-loss from the companion star was included in their study, it was modeled by removal of material from a main sequence star with a constant rate in single-star evolution code. This is an oversimplification and we therefore aim at reexamining the impact of supernova ejecta on a companion star that was modeled consistently in a detailed binary evolution calculation.

Very recently, Pan et al. (2012) studied the impact of SN Ia ejecta on binary companions in the SD scenario with the Eulerian hydrodynamics code FLASH for MS, RG and He star companions. They were able to quantify the amount of contamination with explosion ashes on the companion star by the supernova ejecta in their simulations which might help to identify a companion star even a long time after the explosion.

Based on the prescription of Hachisu et al. (1999b) for the mass growth of CO WDs, and including the possibility of the instability of an accretion disk around the WD on the evolution of binary systems, detailed binary evolution calculations have been performed for about 2400 close WD binaries by Wang et al. (2010, hereafter WLH10). They confirmed that WDs in the WD + MS channel with an initial mass as low as $0.61 M_{\odot}$ can accrete efficiently and reach the Chandrasekhar mass limit. Their calculations also showed that the disk instability could substantially increase the mass-accumulation efficiency for accreting WDs and cause SNe Ia to occur in systems with $\leqslant 2 M_{\odot}$ donor stars. They found that the Galactic SN Ia birth rate from 
the WD + MS channel is $\sim 1.8 \times 10^{-3} \mathrm{yr}^{-1}$ according to their standard model, which can account for $2 / 3$ of the observed SNe Ia.

In this work, we use the same method as WLH10 to carry out consistent binary evolution calculations for the single degenerate MS channel of SNe Ia. With these more realistic companion models, we expand and update the 3D hydrodynamical simulations performed in PRWH08 to investigate the interaction of SN Ia ejecta with MS companion stars. We then explore how the ejecta structure, the stripped mass and the kick velocities of the surviving companion depend on parameters of the progenitor model. Section 2 describes the codes and initial models used in this paper. The SPH impact simulations and numerical results for ten consistent MS companion models are presented in Sect. 3. The comparisons with PRWH08 and some implications of our simulations are discussed in Sect. 4. We summarize and conclude in Sect. 5.

\section{Numerical methods and models}

\subsection{Numerical codes}

We use Eggleton's stellar evolution code (Eggleton 1971, 1972, 1973) to follow the detailed binary evolution of SD progenitor systems. The latest input physics have been implemented by Wang et al. (2009, 2010). Roche lobe overflow (RLOF) is treated in the code as described by Han \& Podsiadlowski (2004). The opacity tables in our code have been compiled by Chen \& Tout (2007) from Iglesias \& Rogers (1996) and Alexander $\&$ Ferguson (1994). We use a typical Population I composition with hydrogen abundance $X=0.70$, helium abundance $Y=0.28$ and metallicity $Z=0.02$. We set the ratio of the typical mixing length to the local pressure scale height, $\alpha=l / H_{\mathrm{P}}$, to 2 , and the convective overshooting parameter, $\delta_{\mathrm{ov}}$, to 0.12 (Pols et al. 1997; Schroder et al. 1997), which roughly corresponds to an overshooting length of $\sim 0.25$ pressure scale heights $\left(H_{\mathrm{P}}\right)$.

In this paper, we start our binary evolution calculations from WD+MS binary systems. In a WD+MS binary system, mass transfer occurs through RLOF when the companion star fills its Roche lobe. If the mass transfer is dynamically stable, the transferred material will form an accretion disk surrounding the WD. This accretion disk can become thermally unstable when the effective temperature in the disk falls below the hydrogen ionization temperature $~ 6500 \mathrm{~K}$ (van Paradijs 1996; Lasota 2001). Here, as a possibility, the effect of the instability of the accretion disk on the evolution of WD + MS binaries has been included to constrain the accretion rate of the WD. If the mass-transfer rate, $\left|\dot{M}_{2}\right|$, is higher than the critical mass-transfer rate for a stable accretion disk, $\dot{M}_{\text {cr,disk }}$, we will assume that the WD accretes smoothly at a rate $\dot{M}_{\text {acc }}=\left|\dot{M}_{2}\right|$. Otherwise, the disk is unstable and the mass-accretion rate of the WD is $\dot{M}_{\text {acc }}=\left|\dot{M}_{2}\right| / d$, where $d$ is the duty cycle, set to 0.1 in this work.

We do not solve the stellar structure equations for the WD star when we construct the structure of the companion star for our simulation. Instead, we adopt the prescription of Hachisu et al. (1999b) for the mass growth of a CO WD by accretion of hydrogen-rich material from a companion. If the mass-accretion rate of the WD, $\dot{M}_{\text {acc }}$, is above a critical value, $\dot{M}_{\mathrm{cr}, \mathrm{WD}}$, we assume that hydrogen burns steadily on the surface of the WD and that the hydrogen-rich material is converted into helium at a rate $\dot{M}_{\mathrm{cr}, \mathrm{WD}}$, while the unprocessed matter is assumed to be lost from the system as an optically thick wind at a mass-loss rate $\dot{M}_{\text {wind }}=\left|\dot{M}_{2}\right|-\dot{M}_{\text {cr,WD. }}$ The critical mass-accretion rate is (Han \& Podsiadlowski 2004)

$\dot{M}_{\mathrm{cr}, \mathrm{WD}}=5.3 \times 10^{-7} \frac{(1.7-X)}{X}\left(M_{\mathrm{WD}} / M_{\odot}-0.4\right) M_{\odot} \mathrm{yr}^{-1}$

where $X$ is the hydrogen mass fraction and $M_{\mathrm{WD}}$ is the mass of the accreting WD. Kato (2011) pointed out that this WD wind case may correspond to the quasi-regular transient supersoft X-ray source (SSS) such as V Sge.

When $\left|\dot{M}_{\text {acc }}\right|$ is smaller than $\dot{M}_{\mathrm{cr}, \mathrm{WD}}$, the following assumptions have been adopted:

1. If $\frac{1}{2} \dot{M}_{\mathrm{cr}, \mathrm{WD}} \leqslant\left|\dot{M}_{\mathrm{acc}}\right| \leqslant \dot{M}_{\mathrm{cr}, \mathrm{WD}}$, it is assumed that there is no mass loss and that hydrogen-shell burning is steady. In this case, before the supernova explosion, the system may be observed as the persistent SSS (Hachisu et al. 2008; Meng \& Yang 2010; Kato 2011).

2. If $\frac{1}{8} \dot{M}_{\mathrm{cr}, \mathrm{WD}} \leqslant\left|\dot{M}_{\mathrm{acc}}\right|<\frac{1}{2} \dot{M}_{\mathrm{cr}, \mathrm{WD}}$, hydrogen-shell burning is unstable, triggering very weak shell flashes, where we assume that the processed mass can be retained. Before the supernova explosion, this case may be observed as recurrent nova of U Sco-type (Hachisu et al. 2008; Meng \& Yang 2010; Kato 2011).

3. If $\left|\dot{M}_{\mathrm{acc}}\right|<\frac{1}{8} \dot{M}_{\mathrm{cr}, \mathrm{WD}}$, hydrogen-shell flashes are so strong that no mass can be accumulated by the WD.

These three cases are accounted for in constructing the setup MS companion stars for our impact simulations. Furthermore, the mass-growth rate of the WD star was linearly interpolated from a grid computed by Kato \& Hachisu (2004). The input physics in our binary evolution calculations is consistent with WLH10 (see also Han \& Podsiadlowski 2004). From our one-dimensional binary evolution calculations, we selected ten progenitor systems with a representative range of orbital periods and initial companion star masses. All resulting models are summarized in Table 1.

For our hydrodynamical simulation of the impact of SN Ia ejecta on their companion stars, we use the GADGET3 code (Springel et al. 2001; Springel 2005). Originally, the GADGET code was intended for cosmological simulations, but it has been modified to make it applicable to stellar astrophysics problems (Pakmor et al. 2012a). By using the initial parameters of the $\mathrm{HCV}^{1}$ scenario of M00, PRWH08 showed that the SPH-based approach is capable of reproducing previous results obtained with a grid-based 2D scheme by M00. This confirms that the SPH approach with the GADGET code captures the main dynamical effects of the supernova impact on its companion star.

\subsection{Basic setup}

In our simulation, we use the same method as PRWH08 to map the one-dimensional profiles of density, internal energy, and nuclear composition of the companion star as obtained from a binary evolution calculation to a particle distribution suitable for the SPH code. Here, the smoothing length is chosen such that a sphere of its radius enclosed 60 neighboring particles. The rest of the basic setup corresponds to that of PRWH08.

To reduce numerical noise introduced by the mapping to ensure that they are in hydrostatic equilibrium before we start the actual simulation, the MS companion stars are relaxed

1 In the HCV scenario, a CO WD accretes hydrogen by RLOF from a lower mass MS secondary. Such a system is formed when a CO WD is left in a close binary orbit by an earlier episode of common envelope evolution in its asymptotic giant branch phase (see Marietta et al. 2000). 
Table 1. Main-sequence companion star models.

\begin{tabular}{cccccccccc}
\hline \hline Model $^{1}$ & $\begin{array}{c}M_{\mathrm{wd}} \\
{\left[M_{\odot}\right]}\end{array}$ & $\begin{array}{c}M_{\mathrm{c}, \mathrm{i}} \\
{\left[M_{\odot}\right]}\end{array}$ & $\begin{array}{c}M_{\mathrm{c}, \mathrm{f}} \\
{\left[M_{\odot}\right]}\end{array}$ & $\begin{array}{c}P_{\mathrm{f}} \\
{[d]}\end{array}$ & $\begin{array}{c}a_{\mathrm{f}} \\
{\left[10^{11} \mathrm{~cm}\right]}\end{array}$ & $a_{\mathrm{f}} / R_{2}$ & $\begin{array}{c}M_{\text {stripped }} \\
{\left[M_{\odot}\right]}\end{array}$ & $\begin{array}{c}v_{\text {kick }} \\
{\left[\mathrm{km} \mathrm{s}^{-1}\right]}\end{array}$ & SNe Ia $^{2}$ \\
\hline ms_20a & 0.70 & 2.00 & 0.74 & 0.98 & 3.72 & 3.06 & 0.181 & 51.01 & disk instability \\
ms_20b & 0.90 & 2.00 & 1.17 & 0.46 & 2.40 & 2.75 & 0.105 & 58.78 & disk instability \\
ms_22a & 0.80 & 2.20 & 1.21 & 0.29 & 1.77 & 2.72 & 0.173 & 105.29 & weak H-shell flash \\
ms_20c & 0.80 & 2.00 & 1.22 & 0.56 & 2.74 & 2.71 & 0.171 & 64.32 & weak H-shell flash \\
ms_24a & 0.90 & 2.40 & 1.40 & 0.33 & 1.95 & 2.63 & 0.172 & 94.95 & stable H burning \\
ms_20d & 1.00 & 2.00 & 1.40 & 0.57 & 2.83 & 2.63 & 0.113 & 53.32 & weak H-shell flash \\
ms_23a & 0.90 & 2.30 & 1.50 & 0.35 & 2.08 & 2.59 & 0.162 & 85.66 & stable H burning \\
ms_24b & 1.00 & 2.40 & 1.88 & 0.37 & 2.25 & 2.46 & 0.116 & 66.91 & stable H burning \\
ms_28a & 1.10 & 2.80 & 2.00 & 0.33 & 2.11 & 2.43 & 0.159 & 84.50 & optically thick wind \\
ms_30a & 1.20 & 3.00 & 2.45 & 0.44 & 2.64 & 2.33 & 0.141 & 65.34 & optically thick wind \\
\hline
\end{tabular}

Notes. $M_{\mathrm{wd}}$ and $M_{\mathrm{c}, \mathrm{i}}$ are the initial masses of WD and its companion at the beginning of mass transfer, respectively. $M_{\mathrm{c}, \mathrm{f}}, P_{\mathrm{f}}, a_{\mathrm{f}}$ and $R_{2}$ denote the final mass of companion star, the orbital period, the binary separation and the radius of secondary at the moment of supernova explosion, respectively. $M_{\text {stripped }}$ and $v_{\text {kick }}$ are the stripped mass and the kick velocity caused by supernova impact. ${ }^{(1)}$ All models have been named with the same way. For example, ms_20(a, b, c, d), the "ms" corresponds to CO WD + MS system, the middle number " 20 " means the $M_{\mathrm{c}, \mathrm{f}}$ is $2.0 M_{\odot}$. The final alphabet "a", "b", "c" or "d" denote the different models with the different $M_{\mathrm{c}, \mathrm{f}}$ but the same $M_{\mathrm{c}, \mathrm{i}}$. ${ }^{\text {(2) }}$ The WD explodes as an SN Ia in the disk instability phase, in the optically thick wind phase, in the stable H-shell burning phase and in the weak H-shell flash phase, respectively.

for $1.0 \times 10^{4} \mathrm{~s}$ (several dynamical timescales). If the relaxation succeeds, the velocities of the particles stay close to zero. Otherwise, we reject the SPH model, and redo the relaxation after adjusting the relaxation parameters (Pakmor et al. 2012a).

The supernova explosion is represented by the W7 model of Nomoto et al. (1984). This model has been shown to provide a good fit to the observational light curves of SNe Ia. Its total explosion energy is $1.23 \times 10^{51} \mathrm{erg}$, and the average velocity of the ejecta is $10^{4} \mathrm{~km} \mathrm{~s}^{-1}$. We place the supernova at a distance to the companion star given by the last separation of the binary system. The impact of the SN Ia ejecta on their binary companions is then simulated for $5000 \mathrm{~s}$, at which point the mass stripped off from the companion star and its kick velocity due to the impact have reached constant values (see Sect. 3.2).

To check the effect of the gravitational field of the WD, we run the impact simulation for the ms_22a model, including a $1.4 M_{\odot}$ WD during relaxation of the companion star. The ms_22a model has the smallest separation (see Table 1) and should therefore be influenced most strongly by the WD. The results show that the companion star is basically not distorted due to the tidal force. The distortion should only be at the percent level in radius anyway, and our spatial resolution in the very outer layers of the star is not sufficient to resolve this. However, the mass in these outer layers is orders of magnitudes smaller than the total stripped mass. Therefore, we run all other simulations in this work ignoring the effect of gravitational field of the WD when the companion stars are relaxed.

\section{Simulations}

In this section, we present the numerical results of our SPH impact simulations for updated MS companion star models. To ensure the reliability of the results from our simulations, we also perform a numerical convergence test for one selected companion star model. We then explore the dependence of stripped mass and kick velocity on the ratios of initial binary orbital separation to companion radius, $a_{\mathrm{f}} / R_{2}$, for a given companion star.

\subsection{Typical evolution}

We discuss the evolution of the model ms_22a (Table 1) as a typical case. Figure 1 shows the snapshots from our SPH impact simulation. We use a total of $6 \times 10^{6} \mathrm{SPH}$ particles to represent the companion star, which corresponds to $\sim 13$ million total SPH particles being used in the simulation. The supernova is represented by the W7 model and set up with an initial separation of $1.77 \times 10^{11} \mathrm{~cm}$ as obtained from the binary evolution simulation.

Figure 1 illustrates the density distribution of the companion star for model ms_22a from the onset of the supernova explosion until the star starts to relax again $(5000 \mathrm{~s})$. At the beginning of the impact simulation, the MS star is in equilibrium. The supernova explodes on the right side of the companion star. After the explosion, the SN ejecta expand freely, approach the companion star from the right side, and a shock wave develops as they hit its surface (see first snapshot). The second snapshot shows the impact $100 \mathrm{~s}$ after the supernova explosion, when the shock starts to propagate through the companion star. At $400 \mathrm{~s}$ (third snapshot), the shock wave reaches its center. The supernova ejecta flow around the companion and a hole forms in them. In the fourth snapshot $(1500 \mathrm{~s})$, the shock wave in the companion has passed the stellar core, and the supernova ejecta are mixed with hydrogen-rich material stripped from the companion star. The last two snapshots show the interaction at $3000 \mathrm{~s}$ and $5000 \mathrm{~s}$ after SN explosion. This is the end of the phase of mass-stripping by the impact; the remnant of the companion star shrinks and relaxes to be almost spherical again. The mass stripped by the impact of the SN ejecta stays constant from this time onwards (see Fig. 2). In Fig. 1, it can be seen that the companion star has moved to left by $\sim 4.5 \times 10^{10} \mathrm{~cm}$ from $0.0 \mathrm{~s}$ to $5000 \mathrm{~s}$ due to the kick caused by the impact.

\subsection{Numerical convergence test}

To ensure reliability of our numerical results, we perform a convergence test. We use model ms_22a and carry out the simulations for different resolutions ranging from $1.07 \times 10^{5}$ to $2.14 \times 10^{7} \mathrm{SPH}$ particles (see Table 2 ). For each resolution, the mass stripped from the companion star at different times after the supernova explosion is calculated (see Fig. 2). The total unbound mass and the kick velocity obtained from the impact $5000 \mathrm{~s}$ after explosion with different resolutions are listed in Table 2. The stripped mass is calculated by summing up the mass of all particles that are not bound to the star any more, but were part of the star in the initial setup. To determine whether or not a particle 


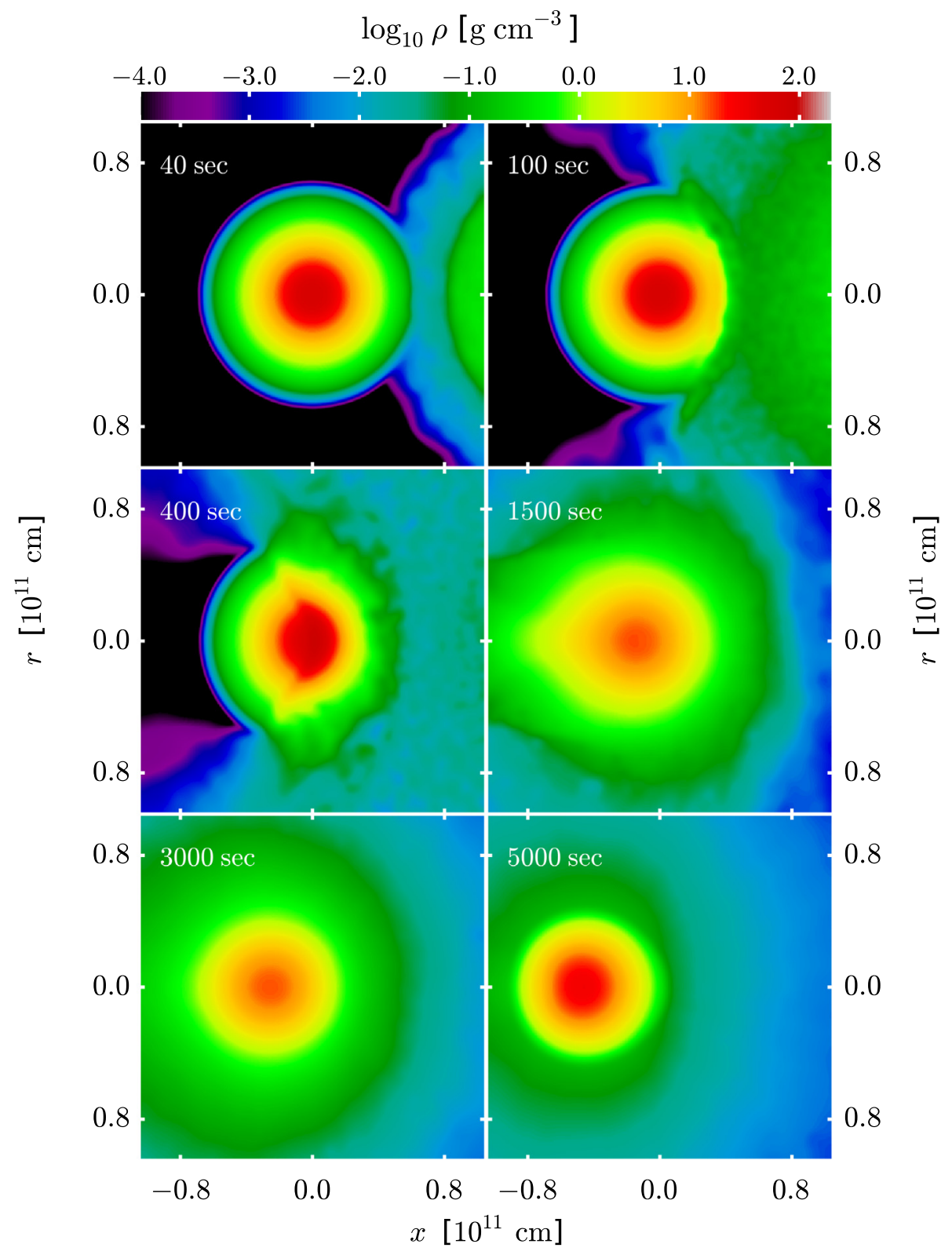

Fig. 1. Time evolution of density distribution in the impact simulation with model ms_22a. For visualization (not for the simulation, though), we use cylindrical coordinates. The radial coordinate is averaged over angle accounting for the intrinsic symmetry of the star. The logarithm of density is color-coded. is bound to the star, we calculated the sum of the kinetic energy (positive) and potential energy (negative) for each particle. If the total energy is positive, the particle is not bound. Note that the center-of-mass motion of star is subtracted when calculating the kinetic energy for each particle.

Figure 2 shows that the mass loss decreases when the number of the SPH particles used in the simulations increases (see also Fig. 3). However, it is numerically well converged for more than about 8 million SPH particles in the simulation (this corresponds about 4 million particles in the companion star). The difference in stripped mass between simulations with $8.56 \times 10^{6}$ (the short dashed line) and $1.28 \times 10^{7}$ (the dotted line) SPH particles is smaller than $2 \%$, the difference between $1.28 \times 10^{6}$ and $2.14 \times 10^{7}$ (the narrow dash-dotted line) SPH particles is less than $1 \%$ (see Table 2). After $3000 \mathrm{~s}$, the amount of unbound mass becomes constant in good approximation. The density distribution of the companion star corresponding to different resolutions is shown in Fig. 4. There are also no morphological differences among the simulations with $8.56 \times 10^{6}, 1.28 \times 10^{7}$ and $2.14 \times 10^{7}$ SPH particles (the last three snapshots in Fig. 4). Therefore, we conclude that it is sufficient to represent the companion stars with about 6 million SPH particles in our SPH impact simulations.

\subsection{Results}

Based on a selection of ten realistic MS companion star models we simulate the interaction of supernova ejecta with their binary companions. The initial parameters of all binary systems used in this paper are listed in Table 1.

The amount of hydrogen-rich material stripped from the surface of the companion stars, $M_{\text {stripped }}$, and the kick velocities caused by the supernova impact, $v_{\text {kick}}$, are also shown in Table 1 (see also Fig. 5). The stripped masses range from 0.11 to $0.18 M_{\odot}$ and we measure kick velocities between 51 and $105 \mathrm{~km} \mathrm{~s}^{-1}$. Note that this kick velocity is defined as the center of mass velocity of all particles bound to the companion star $5000 \mathrm{~s}$ after the explosion of the supernova.

The largest stripped mass of our simulations is found for model ms_20a $\left(0.18 M_{\odot}\right)$. Further analysis shows that the hydrogen in the center of this companion star has already been mostly depleted at the time the white dwarf explodes in a supernova, 


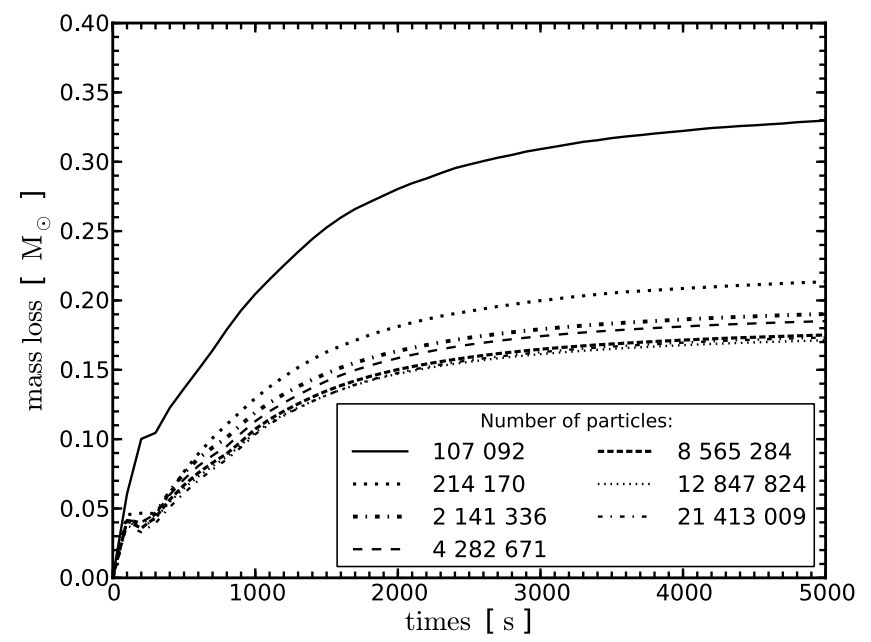

Fig. 2. Temporal evolution of the mass loss from the companion star for simulations with different numbers of SPH particles. Note that the number of particles gives the total of particles in the simulation (both supernova and companion star).

Table 2. Resolution test for ms_22a model.

\begin{tabular}{lcccc}
\hline \hline$N_{\text {star }}$ & $N_{\text {total }}$ & $\begin{array}{c}m_{\text {particle }} \\
{\left[M_{\odot}\right]}\end{array}$ & $\begin{array}{c}M_{\text {stripped }} \\
{\left[M_{\odot}\right]}\end{array}$ & $\begin{array}{c}v_{\text {kick }} \\
{\left[\mathrm{km} \mathrm{s}^{-1}\right]}\end{array}$ \\
\hline 50000 & 107092 & $2.41 \times 10^{-5}$ & 0.329 & 138.17 \\
100000 & 214170 & $1.21 \times 10^{-5}$ & 0.215 & 123.92 \\
1000000 & 2141336 & $1.21 \times 10^{-6}$ & 0.190 & 111.75 \\
2000000 & 4282671 & $6.04 \times 10^{-7}$ & 0.185 & 108.90 \\
4000000 & 8565284 & $3.02 \times 10^{-7}$ & 0.175 & 106.54 \\
6000000 & 12847824 & $2.01 \times 10^{-7}$ & 0.173 & 105.29 \\
10000000 & 21413009 & $1.21 \times 10^{-7}$ & 0.173 & 105.31 \\
\hline
\end{tabular}

Notes. $N_{\text {star }}$ and $N_{\text {total }}$ are the number of particles used to represent the companion star and the binary system, respectively. All particles have the same mass $m_{\text {particle }}$.

and its outer layers already begin to expand. This moves the star on its evolutionary track in the Hertzsprung-Russell (H-R) diagram towards the giant phase. The density profile of companion star ms_20a is shown in next section. Compared with other main sequence stars in our sample, this star has a very large radius and a higher density in the core. The shrinking of its inner core and the subsequent expansion of the outer layers make its envelope less bound. This explains why more hydrogen-rich material is stripped off when the supernova blast wave hits the companion star in this model.

For a given companion star model, we investigate the dependence of stripped mass, $M_{\text {stripped }}$, and kick velocity, $v_{\text {kick}}$, on the ratio of orbital separation to companion star radius, $a_{\mathrm{f}} / R_{2}$. All parameters but the orbital separation are kept constant (it means that we only change the value of the separation, $a_{\mathrm{f}}$, for the same companion model artificially). Figure 5 shows the stripped mass and kick velocity versus $a_{\mathrm{f}} / R_{2}$ for models ms_22a, ms_24a and ms_28a. For a given companion star, we find a similar relationship as previous studies (Marietta et al. 2000; Meng et al. 2007; Pakmor et al. 2008; Pan et al. 2012). The dependence of the stripped mass on this ratio follows a power law to good approximation (see Fig. 5):

$M_{\text {stripped }}=a_{0} \cdot\left(\frac{a_{\mathrm{f}}}{R_{2}}\right)^{v} \quad M_{\odot}$.

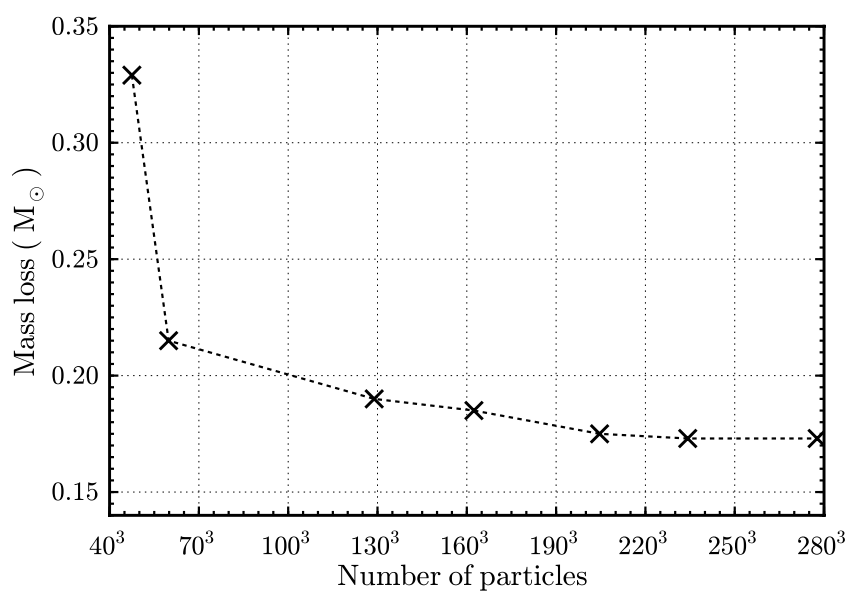

Fig. 3. Mass loss vs. different number of SPH particles used in ms_22a model simulations.

Likewise, the dependence of the kick velocity, $v_{\text {kick }}$ on $a_{\mathrm{f}} / R_{2}$ can be fitted by a power law (see Fig. 5)

$v_{\text {kick }}=a_{1} \cdot\left(\frac{a_{\mathrm{f}}}{R_{2}}\right)^{\mu} \mathrm{cm} \mathrm{s}^{-1}$.

Here, $a_{0}$ and $a_{1}$ are two fitting constants, which, however, are not unique but depend on the companion star models. The parameters $v$ and $\mu$ are the corresponding power law indices. Values for these fitting parameters are listed in Table 3. For comparison, we also show the result from model rp3_20a of PRWH08. Comparing our models with PRWH08, all simulations follow a power law of some kind, but we do not obtain the same fitting parameters. This implies the importance of the structure of the companion star for the stripped mass and kick velocity in our impact simulations. Furthermore, we put all models used in this paper and the PRWH08 models together as a whole sample to examine the effect of $a_{\mathrm{f}} / R_{2}$. Figure 6 shows the dependence of the stripped mass and kick velocity on the parameter, $a_{\mathrm{f}} / R_{2}$, for all these models. However, we do not find a power law relation is still holding in Fig. 6. Therefore, again, it indicates the results of our impact simulations are also dependent on the details of the structure of the companion stars due to the history of mass transfer.

\section{Discussion}

\subsection{Comparison with previous studies}

Wheeler et al. (1975) derived a simple analytic formula for the calculation of stripped mass, and their results were confirmed by some of the early numerical simulations (see Marietta et al. 2000). Here, we also estimate the stripped mass using their analytic method based on our MS companion models (see Table 4). As shown in Table 4, the order of magnitude of stripped mass predicted by the analytic estimate agrees with our results, but is usually overestimated by the analytic formula compared to the result obtained from our simulations, with deviations ranging from $5 \%$ to $43 \%$.

Using a $1.0 M_{\odot}$ solar-like companion star model, Marietta et al. (2000) found about $0.15 M_{\odot}$ hydrogen-rich material to be stripped from the surface of MS companion by the impact of the supernova explosions. The mass of stripped hydrogen-rich material in our simulation is consistent with their results. Note that compared to the study of PRWH08 our simulations bring the 


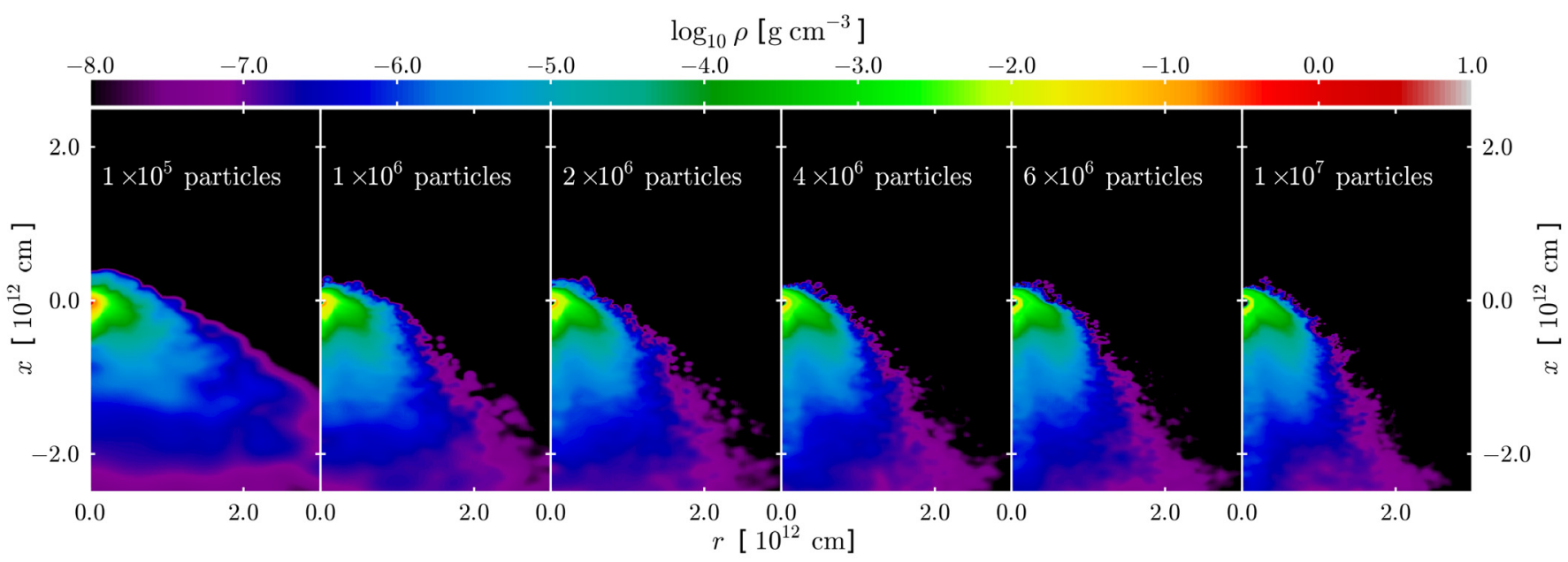

Fig. 4. Density distribution of the companion star in the ms_22a model at $3000 \mathrm{~s}$ after supernova explosion with different resolutions. Density is color-coded logarithmically.
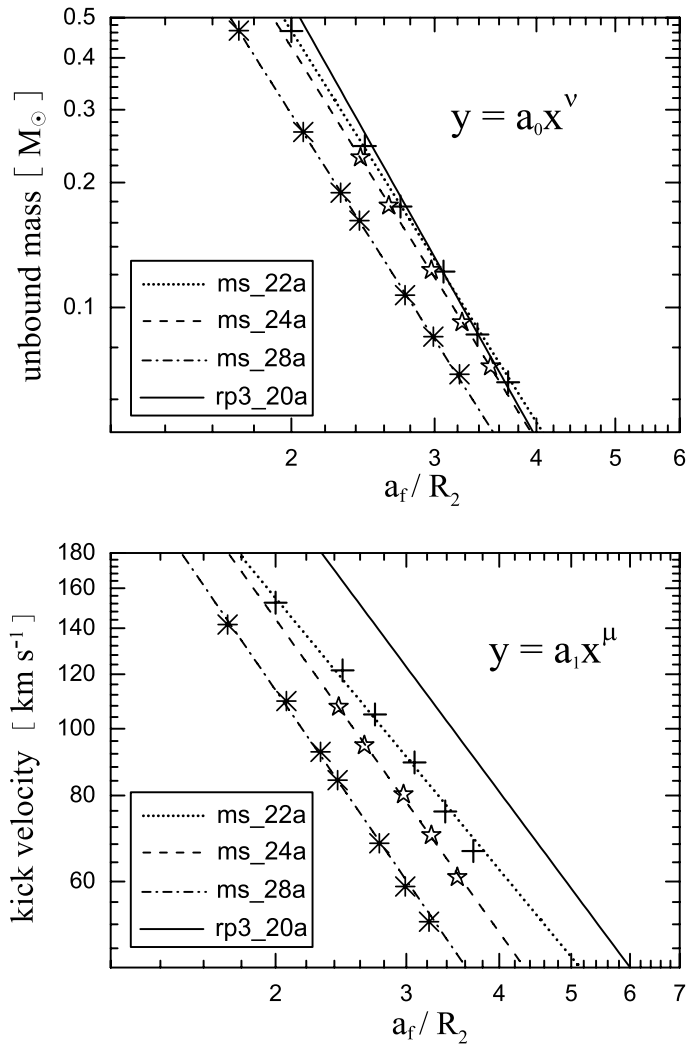

Fig. 5. Final stripped mass and kick velocity vs. the ratio of orbital separation to the companion radius for different companion models. The data are fitted by using the power law of Eqs. (2) or (3) in this paper. The solid line corresponds to the rp3_20a model of PRWH08. Note that we use logarithmic coordinates here. All fitting parameters are given in Table 3 .

results back to the original work of Marietta et al. (2000) only by chance - the setups of the companion stars are very different. While we construct our companion star models from consistent binary evolution calculations, their model ignores the effect of mass transfer on the structure of the companion star altogether. Although PRWH08 included such an effect in the construction of their companion stars, it was not done in a consistent binary evolution calculation.
Table 3. Fitting parameters of Eqs. (2) and (3).

\begin{tabular}{lcccc}
\hline \hline Models & $a_{0}$ & $v$ & $a_{1}$ & $\mu$ \\
\hline ms_22a & 4.092 & -3.137 & $3.84 \times 10^{7}$ & -1.309 \\
ms_24a & 3.786 & -3.156 & $4.11 \times 10^{7}$ & -1.509 \\
ms_28a & 2.503 & -3.095 & $3.38 \times 10^{7}$ & -1.573 \\
rp3_20a & 6.105 & -3.489 & $6.05 \times 10^{7}$ & -1.450 \\
\hline
\end{tabular}
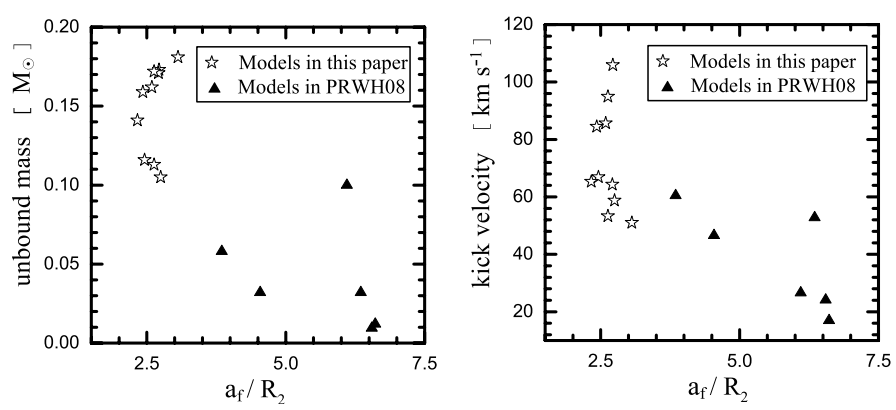

Fig. 6. Final stripped mass and kick velocity versus the ratio of separation to the radius of companion star, $a_{\mathrm{f}} / R_{2}$, for different companion star models. The pentacles and filled triangles denote the models simulated in this paper and models in PRWH08, respectively.

Table 4. Stripped mass.

\begin{tabular}{lccccc}
\hline \hline Model & $\begin{array}{c}M_{\mathrm{c}, \mathrm{f}} \\
{\left[M_{\odot}\right]}\end{array}$ & $a_{\mathrm{f}} / R_{2}$ & $\begin{array}{c}\Delta M^{1} \\
{\left[M_{\odot}\right]}\end{array}$ & $\begin{array}{c}\Delta M^{2} \\
{\left[M_{\odot}\right]}\end{array}$ & $\begin{array}{c}\text { Difference } \\
{[\%]}\end{array}$ \\
\hline ms_20a & 0.74 & 3.06 & 0.18 & 0.19 & 5 \\
ms_20b & 1.17 & 2.75 & 0.11 & 0.14 & 27 \\
ms_22a & 1.21 & 2.72 & 0.17 & 0.18 & 6 \\
ms_20c & 1.22 & 2.71 & 0.17 & 0.14 & 18 \\
ms_24a & 1.40 & 2.63 & 0.17 & 0.19 & 12 \\
ms_20d & 1.40 & 2.63 & 0.11 & 0.15 & 36 \\
ms_23a & 1.50 & 2.59 & 0.16 & 0.19 & 19 \\
ms_24b & 1.88 & 2.46 & 0.12 & 0.17 & 42 \\
ms_28a & 2.00 & 2.43 & 0.16 & 0.21 & 32 \\
ms_30a & 2.45 & 2.33 & 0.14 & 0.20 & 43 \\
\hline
\end{tabular}

Notes. ${ }^{(1)}$ Numerical calculation of stripped mass in our simulations. (2) Stripped mass analytically estimated by using the method of Wheeler et al. (1975). Here, we directly use the density profiles of companion models from the 1D stellar evolution calculations. 


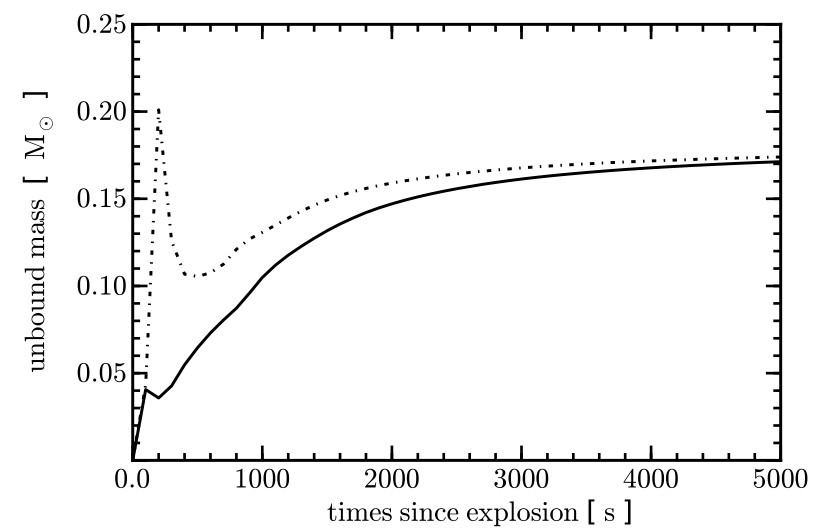

Fig. 7. Unbound mass vs. simulation time in ms_22a model. The solid line shows the total mass of all particles with a total energy (kinetic plus potential energy) larger than zero. The dash-dotted line also includes the internal energy in the sum.

The amount of the unbound mass in our work is also consistent with the new multi-dimensional adaptive mesh refinement simulations of Pan et al. (2012). But, again, they did not follow the full binary evolution but used initial conditions with a constant mass-loss rate when constructing their MS companion star models with the MESA code of Paxton et al. (2011). Therefore, the agreement can be regarded as coincidental, too. Pan et al. (2012) argue that the mass stripping is dominated by ablation in their simulations for the MS companion. They used a slightly different criterion for the unbound mass in which they included the internal energy in addition to the potential and kinetic energy. This does not make an effect for our simulations because we are mainly interested in the unbound mass at late enough times when the internal energy is negligible to the kinetic energy. Figure 7 shows the unbound mass of a companion star as a function of time after the supernova explosion in our SPH simulation. Whether or not a particle is unbound is decided by summing its potential energy, kinetic energy and internal energy (or without the internal energy, which corresponds to the solid line in Fig. 7). Already $5000 \mathrm{~s}$ after the explosion most of the internal energy deposited by the impact has been converted into kinetic energy. Therefore, as in PRWH08, we neglect the internal energy when we flag particles as bound or unbound at late times in our simulation.

In our study we find that a minimum of $0.1 M_{\odot}$ of hydrogenrich material being stripped from the companion star. This is significantly larger than the $0.035 M_{\odot}$ for stripped hydrogen found by Meng et al. (2007). This might be caused by their oversimplified description of the interaction physics, e.g. the effect of the shock formed between the supernova ejecta and the companion star was not calculated in their analytic model. Recently, Pan et al. (2012) argued that since they neglected the mass loss due to the ablation from the hot surface of the companion star, Meng et al. (2007) underestimate the final unbound mass.

PRWH08 found stripped masses in the range from $0.01 M_{\odot}$ to $0.06 M_{\odot}$, which is very close the observational limit obtained by Leonard (2007), but significantly lower than the values we find for the models presented here. To determine the origin of this difference, we select two models, ms_20c and ms_22a (see Table 1), in comparison to the models rp3_20a and rp3_20b of PRWH08. The density profiles of these four models at the moment of the explosion of the supernova are shown in Fig. 8. Models ms_22a and rp3_20b have the same radius of $0.65 \times 10^{11} \mathrm{~cm}$. Although there are some small differences in

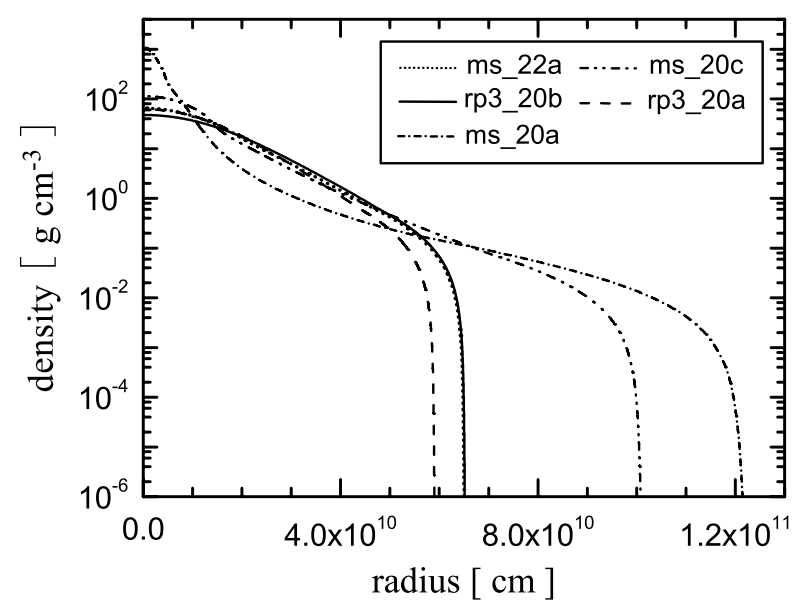

Fig. 8. Initial density profiles of five companion star models at the onset of the SN Ia explosion. Model rp3_20a (dashed line) and rp3_20b (solid line) are from PRWH08.

the density profiles in the inner cores, the outer layers are nearly identical. In our simulations, only the properties of outer layers of the companions can significantly affect the results of the interaction between supernova ejecta and companion star. Therefore, we chose these two models to carry out a comparison. PRWH08 had set up the binary with a separation of $4.26 \times 10^{11} \mathrm{~cm}$ for their Model rp3_20b. They found a stripped mass of $0.01 M_{\odot}$ and a kick velocity of $24.1 \mathrm{~km} \mathrm{~s}^{-1}$. Our model ms_22a, however, has an orbital separation at the time of the explosion of $1.77 \times 10^{11} \mathrm{~cm}$ only. Running this model we obtain a significantly larger stripped mass of $0.17 M_{\odot}$ and a higher kick velocity of $105.29 \mathrm{~km} \mathrm{~s}^{-1}$. This is to be expected for changing the initial separation, as discussed above. Furthermore, we calculate the stripped mass and kick velocity with the same separation $4.26 \times 10^{11} \mathrm{~cm}$ as for Model rp3_20b by using the power law relation of Eqs. (2) and (3) for our Model ms_22a. We find a stripped mass of $0.01 M_{\odot}$ and kick velocity of $33.02 \mathrm{~km} \mathrm{~s}^{-1}$, which is excellent agreement with Model rp3_20b. Therefore, excluding the density effect, the orbital separation at the time of the explosion is the primary factor to cause the difference between the simulations of the two models ms_22a and rp3_20b.

The difference in orbital separations originates from different treatments of the progenitor evolution. The binary systems we examine here fill their Roche-lobe at the time of the explosion. This fixes the orbital separations of the binary systems. Instead of detailed binary evolution calculations, PRWH08 directly took parameters from the study of Ivanova \& Taam (2004) to mimic the effect of the binary evolution phase. Ivanova \& Taam (2004) analyzed the evolution of possible SN Ia progenitor systems consisting of a WD and an evolved MS star. In PRWH08, all values adopted by model rp3_20a come from a WD+MS binary system evolved by Ivanova \& Taam (2004) with initial WD mass $M_{\mathrm{WD}}=0.8 M_{\odot}$, companion mass $M_{\mathrm{d}, \mathrm{i}}=2.0 M_{\odot}$ and orbital period $P_{\mathrm{i}}=1 \mathrm{~d}$ (Ivanova \& Taam 2004). These parameters have been presented in Table 2 of PRWH08. For comparison, we set up our new Model ms_20c with the same initial binary parameters $\left(M_{\mathrm{WD}}=0.8 M_{\odot}, M_{\mathrm{d}, \mathrm{i}}=2.0 M_{\odot}\right.$ and $\left.P_{\mathrm{i}}=1 \mathrm{~d}\right)$ and carry out the fully detailed binary evolution calculation to construct the structure of companion star. Properties of Model ms_20c are listed in Table 1. The final orbital separation at the moment of the supernova explosion is $2.74 \times 10^{11} \mathrm{~cm}$, which is very close to the $2.68 \times 10^{11} \mathrm{~cm}$ of Model rp3_20a by PRWH08. Moreover, the final companion masses agree very well $\left(1.22 M_{\odot}\right.$ for our 
Z. W. Liu et al.: 3D simulations of interaction between SN Ia ejecta and their MS companions
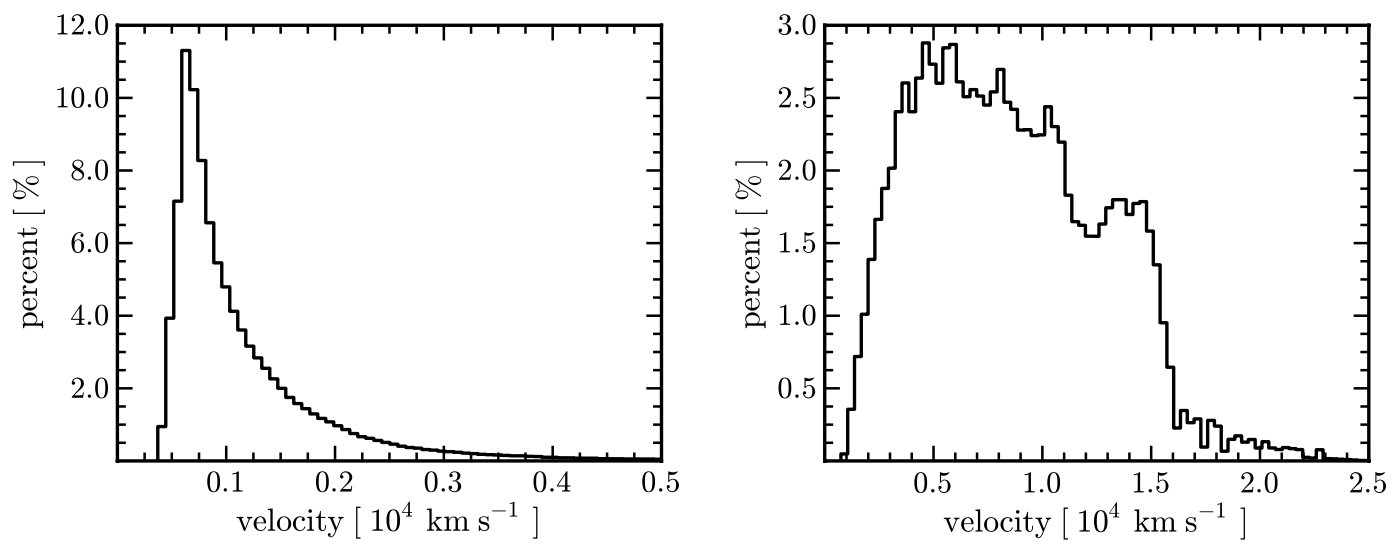

Fig. 9. Final velocity distribution of the stripped hydrogen-rich material that originally belonged to the companion star (left panel) and supernova ejecta (right panel) $5000 \mathrm{~s}$ after the supernova explosions for model ms_22a.

Model ms_20c versus $1.17 M_{\odot}$ for Model rp3_20a). However, Fig. 8 shows that the ms_20c model has a larger radius compared to Model rp3_20a. Therefore, it is not surprising that the impact simulation for this model leads to a stripped mass of $0.171 M_{\odot}$ (see Table 1 ), while only $0.032 M_{\odot}$ were stripped in the rp3_20a model. Since our Model ms_20c has a larger radius, it has a less bound envelope that can be stripped away more easily. Moreover, the larger radius causes an extended interaction area that also leads to a slightly larger kick velocity of $64.32 \mathrm{~km} \mathrm{~s}^{-1}$ compared with the $46.6 \mathrm{~km} \mathrm{~s}^{-1}$ of Model rp3_20a. Finally, in our model, a larger conical hole is created in the supernova debris behind the companion star. Without the effect of orbital separation, the more compact structure of the companion star significantly reduces the stripped mass. Thus, the degree of compactness of a companion star, especially the compactness of its outer layer is very important to determine the influence of the impact of the SN Ia ejecta on its binary companion star.

What causes this difference of companion star structures between the models ms_20c and rp3_20a? PRWH08 computed their companion star models by constantly removing mass while they evolved a single main sequence star. They did not consider the detailed mass-transfer processes in a binary system. In their one-dimensional stellar evolution, the mass loss proceeds rather rapidly: the duration of the mass transfer is about a factor of 10 less than the Kelvin-Helmholtz timescale of their stars. Although they mimicked the total mass loss and the total time of mass loss from Ivanova \& Taam (2004) when constructing their companion star models, this does not lead to realistic companion star structures such as obtained from consistent binary evolution. The result are more compact objects and the binary systems of PRWH08 are characterized by very large values of the parameter, $a_{\mathrm{f}} / R_{2}$, since they used less evolved companion stars to start the mass accretion phase from, which were too compact to actually fill their Roche-lobe (see Fig. 6). In contrast, RLOF is taken into account for the mass transfer of a binary system in our detailed binary evolution calculations, and we also consider the process of WD accretion by including the optically thick wind model of Hachisu et al. (1999b). The structures of companion stars are properly adjusted due to the detailed modeling of mass transfer in the binary system. Consequently, our work presents an update to the PRWH08 study with more realistic companion star models.

In our SPH impact simulations, the stripped mass and the kick velocity depend sensitively on the ratio of the orbital separation to the radius of companion star, $a_{\mathrm{f}} / R_{2}$. More compact companion stars with a larger orbital separation lead to a significant

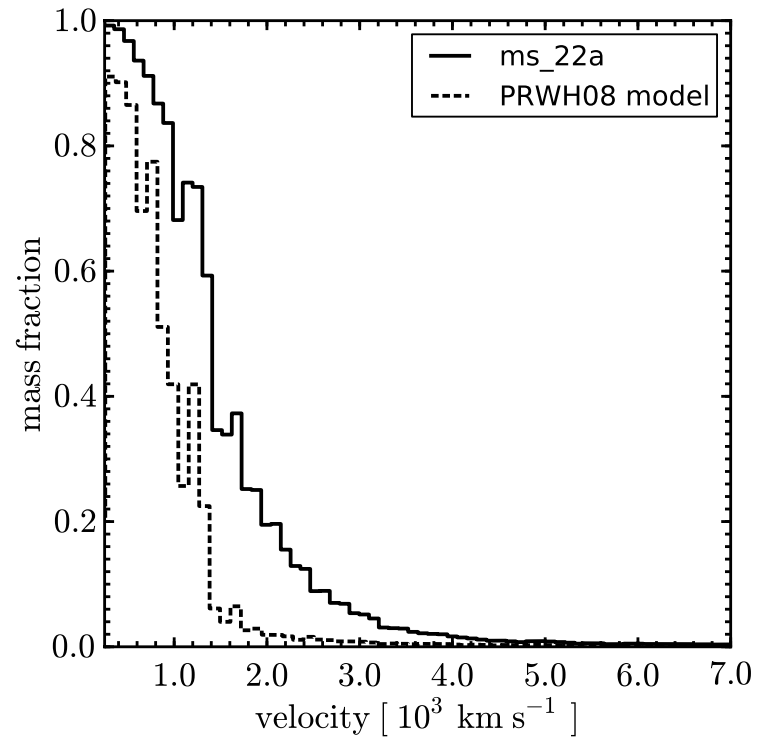

Fig. 10. Fraction of the material stripped from the companion in velocity space for ms_22a model $5000 \mathrm{~s}$ after the supernova explosion relative to the total contaminated supernova ejecta. The dashed line corresponds to the PRWH08 model.

reduction of the amount of stripped hydrogen-rich material. However, $a_{\mathrm{f}} / R_{2}$ is not the only parameter to determine the outcome of the supernova impact, the companion structure is also important (see Fig. 6).

\subsection{Distribution of the stripped material in velocity space}

The hydrogen-rich material stripped from the companion star is mixed into the supernova ejecta. Figure 9 shows the velocity distribution of the hydrogen-rich material stripped from the companion star (left panel) and the supernova ejecta (right panel). Most of the stripped material is concentrated at velocities below $\sim 800 \mathrm{~km} \mathrm{~s}^{-1}$, which is much slower than the typical velocities of the supernova ejecta of $\sim 10000 \mathrm{~km} \mathrm{~s}^{-1}$ (Chugai 1986; Marietta et al. 2000), placing it at the very center of the ejecta.

In Fig. 10 we plot the mass fraction of material stripped from the companion star in velocity space (all bound material has been cut out). We also show the result of PRWH08 (dashed line) for comparison. In the low velocity region, the mass fraction of the stripped material is very high and it dominates over the original 


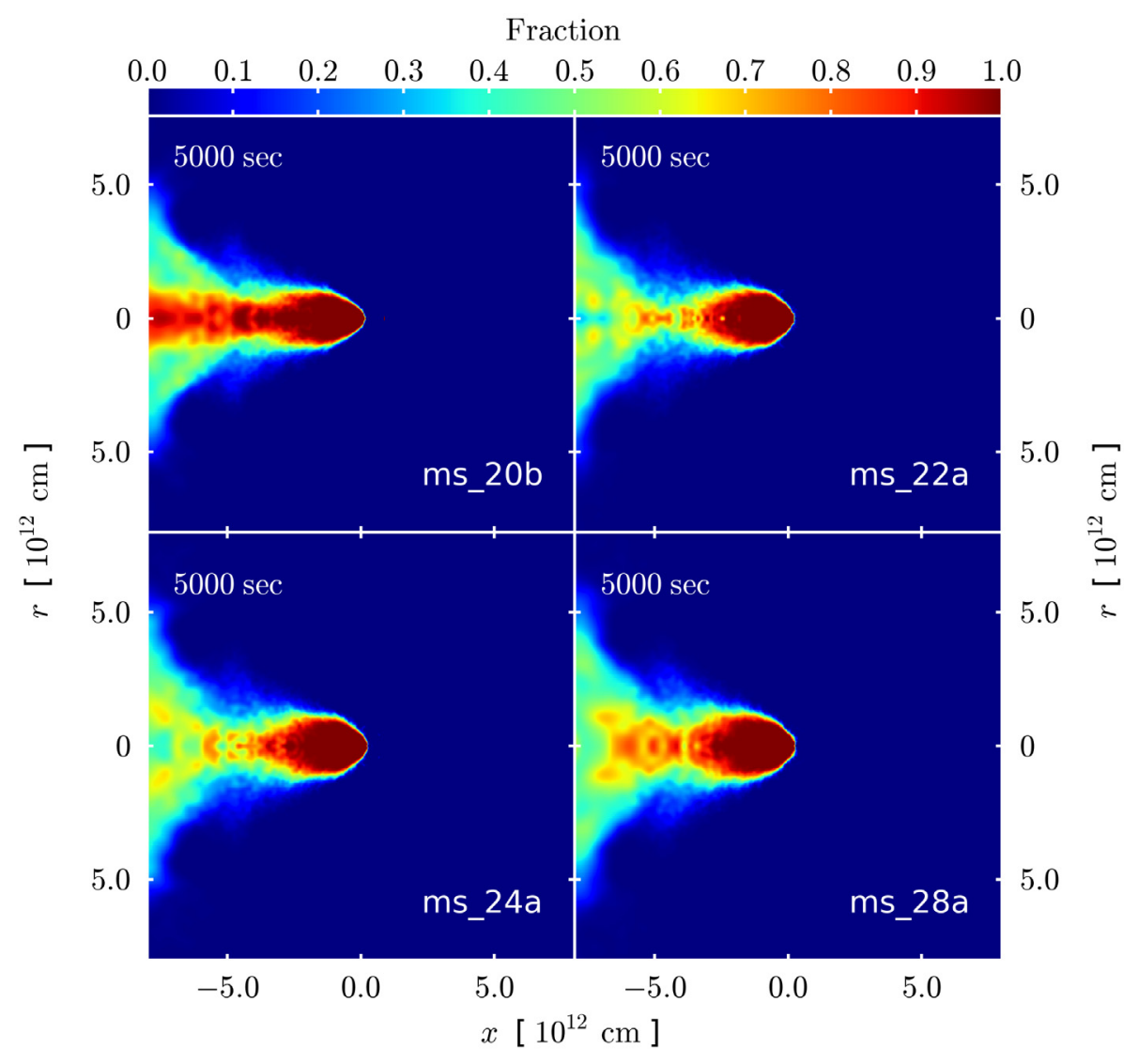

Fig. 11. Relative fraction of material that belonged originally to the companion with respect to the total amount of material. The red and blue color correspond to companion and supernova material, respectively. supernova material. But this fraction sharply decreases as the velocity becomes larger than $10^{3} \mathrm{~km} \mathrm{~s}^{-1}$. Clearly, the supernova ejecta dominate at high velocities. However, some stripped material is present also in the outer ejecta as already noted by M00. They argued that the presence of stripped material at high velocities implies that traces of hydrogen from the companion are swept up in the oxygen and silicon layer of the supernova ejecta. They also argued that given the upper limits on the hydrogen abundance from SN Ia observations near maximum light, this may provide a criterion for discriminating between SN Ia progenitor scenarios (M00). Figure 11 illustrates the relative amount of material that originally belonged to the companion with respect to the total amount of material for different companion star models. This figure shows how the supernova ejecta are mixed with the material stripped from the surface of the companion star. Most of the stripped material, however, will only become visible at very late times when the ejecta are mostly transparent and it is possible to see very deep into the center of the ejecta.

Figure 12 shows how the spatial distribution of stripped material evolves with time for model ms_20c. The left column shows both, the SN Ia and companion star at 1000 s, $3000 \mathrm{~s}$ and $5000 \mathrm{~s}$, respectively. The right column shows the material originally belonging to the supernova only - all companion material has been cut out. We see that the supernova ejecta are significantly affected by the companion star when the supernova impacts the binary companion. The interaction creates a conical hole in the supernova debris with an opening angle of about $50^{\circ}$ (see the right snapshot at $1000 \mathrm{~s}$ of Fig. 12). Comparing with PRWH08, our simulation shows a larger cone-like hole behind the companion star. This is not surprising, because at the same initial separation our companion star has a larger radius than the models used in PRWH08. Additionally, Fig. 12 shows that the material is shocked at the companion star which leads to the formation of a bow shock. Ejecta passing through the shock are heated and compressed into a thin shell, and their velocity vector is redirected (Kasen 2010).

\subsection{Detection of hydrogen}

In our simulations, we find that the supernova impact strips off $0.11 M_{\odot}$ to $0.18 M_{\odot}$ of hydrogen-rich material from the companion star (see Table 1). This is far more than the most stringent upper limit of $0.01 M_{\odot}$ which Leonard (2007) derived from the non-detection of $\mathrm{H}_{\alpha}$ emission in late time spectra. Therefore our results might challenge the SD scenario if the systems studied here are representative and the objects, from which the observational constraints were derived, originate from SD progenitors.

However, it is important to note that the model of Mattila et al. (2005) which was employed by Leonard (2007) to obtain the upper limit mentioned above is quite simple. Moreover, most of the stripped hydrogen in our models ends up at velocities below $10^{3} \mathrm{~km} \mathrm{~s}^{-1}$ so that it is confined to the innermost part of the explosion ejecta which are usually rich in iron-group elements. Whether or not $\mathrm{H}_{\alpha}$ emission will be detectable under these conditions, is a highly complex question which can only be answered by performing sophisticated radiative transfer simulations on the abundance structure of our explosion models. This is beyond the scope of the current paper.

Note also that there are some possibilities to reduce the amount of stripped hydrogen. In our detailed binary evolution calculations, we adopt the prescription of Hachisu et al. (1999a) for the mass growth of a CO WD by accretion of hydrogen-rich material from its companion. According to this model unprocessed matter is assumed to be lost from the binary system due to an optically thick wind if the mass accretion rate exceeds a critical value of $\dot{M}_{\mathrm{cr}, \mathrm{WD}}=5.3 \times 10^{7}(1.7 / X-1)\left(M_{\mathrm{WD}}-0.4\right) M_{\odot} \mathrm{yr}^{-1}$ 


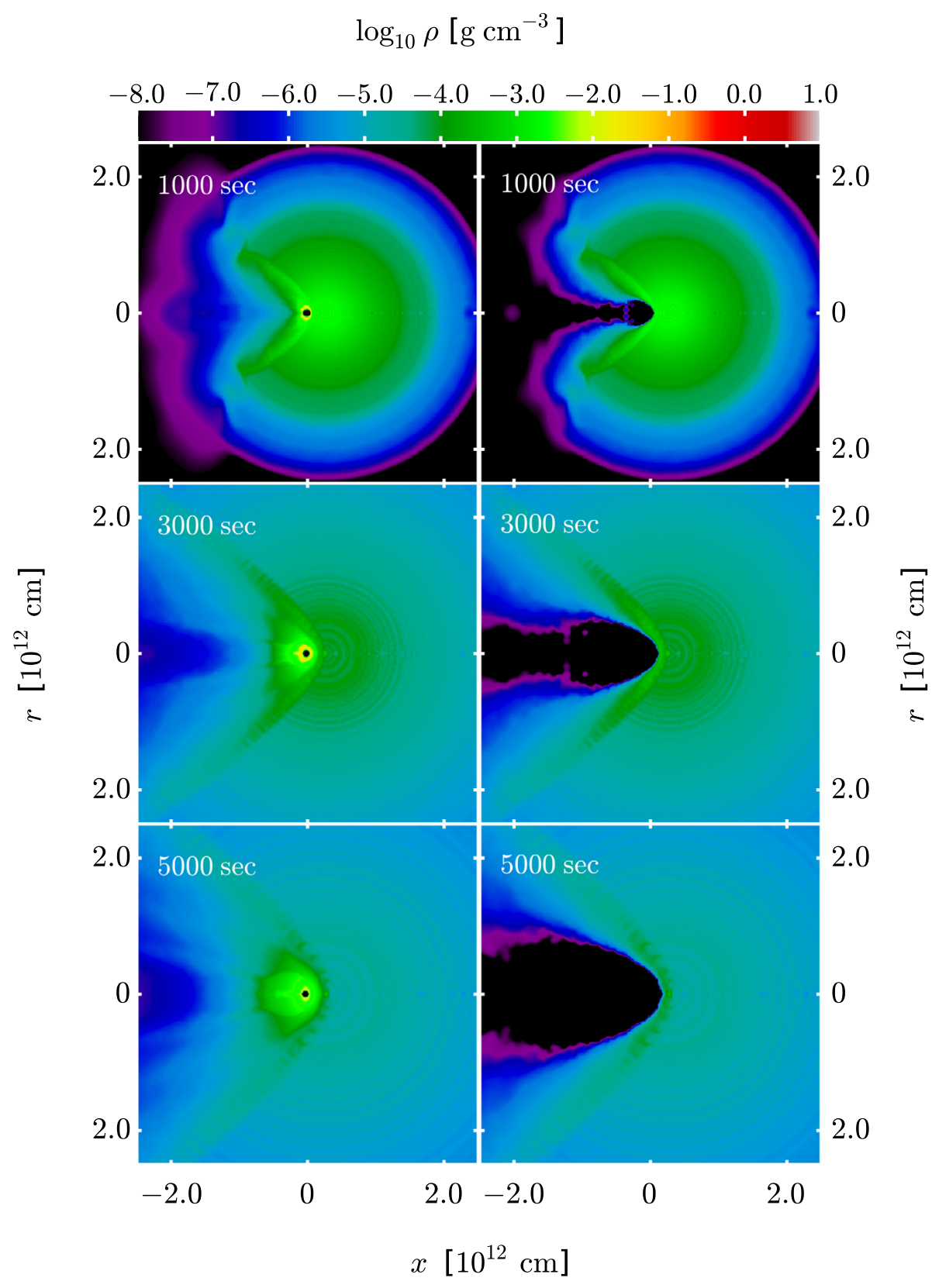

Fig. 12. Density distributions of all material (left panel) and the supernova ejecta (right panel) at $1000 \mathrm{~s}, 3000 \mathrm{~s}$ and $5000 \mathrm{~s}$ after the explosion for model ms_20c. The logarithm of density is color-coded. (here, $X$ is the hydrogen mass fraction and $M_{\mathrm{WD}}$ the mass of the accreting WD). Hachisu et al. (1999a, 2008) proposed that this optically thick wind will strip off parts of the outer layer of the companion star. This "mass-stripping" effect is currently neglected in our simulations. However, it could reduce the amount of hydrogen in the $\mathrm{SN}$ ejecta since the mass-stripping reduces the companion size and therefore also the mass lost in the supernova impact.

Another possibility to reduce the amount of stripped hydrogen arises if the MS companion in the binary has a helium-rich envelope (Pan et al. 2010, find the stripped helium mass to be consistent with observational constraints in this case). Such a system is possible if a massive primary undergoes a first RLOF during its red giant phase and the resulting helium star experiences a second RLOF episode during core helium burning (Hachisu et al. 1999a,b).

Finally, we note that also the recently proposed "spin-up and spin-down" model (Justham 2011; Di Stefano et al. 2011; Hachisu et al. 2012) is likely to reduce hydrogen stripping.

\subsection{Surviving companion stars}

In the $\mathrm{SD}$ scenario of $\mathrm{SNe}$ Ia, the companion star survives the supernova explosion. Because the interaction with the SN Ia ejecta and its orbital velocity at the time of the explosion, its spatial velocity might distinguish it from stars in its neighborhood. In our simulation, the kick velocity reaches values from $51 \mathrm{~km} \mathrm{~s}^{-1}$ to $105 \mathrm{~km} \mathrm{~s}^{-1}$, which is comparable to the orbital velocity of 96-281 $\mathrm{km} \mathrm{s}^{-1}$. Thus, the spatial velocity of the remnant star, $v_{\text {spatial }}=\sqrt{v_{\text {kick }}^{2}+v_{\text {orb }}^{2}}$, ranges from $108 \mathrm{~km} \mathrm{~s}^{-1}$ (model ms_20a) to $287 \mathrm{~km} \mathrm{~s}^{-1}$ (model ms_30a). Ruiz-Lapuente et al. (2004) showed that Tycho $G$ star has a spatial velocity of $136 \mathrm{~km} \mathrm{~s}^{-1}$, which is located in the range of spatial velocities in our models.

Furthermore, a surviving companion star will be strongly affected by the impact of an SN Ia and show distinguishing properties. The identification of a surviving companion stars in historical supernova remnants is a promising method to test progenitor models of SNe Ia. Figure 13 shows the evolution of a remnant companion star up to $5000 \mathrm{~s}$ after the supernova explosion for 

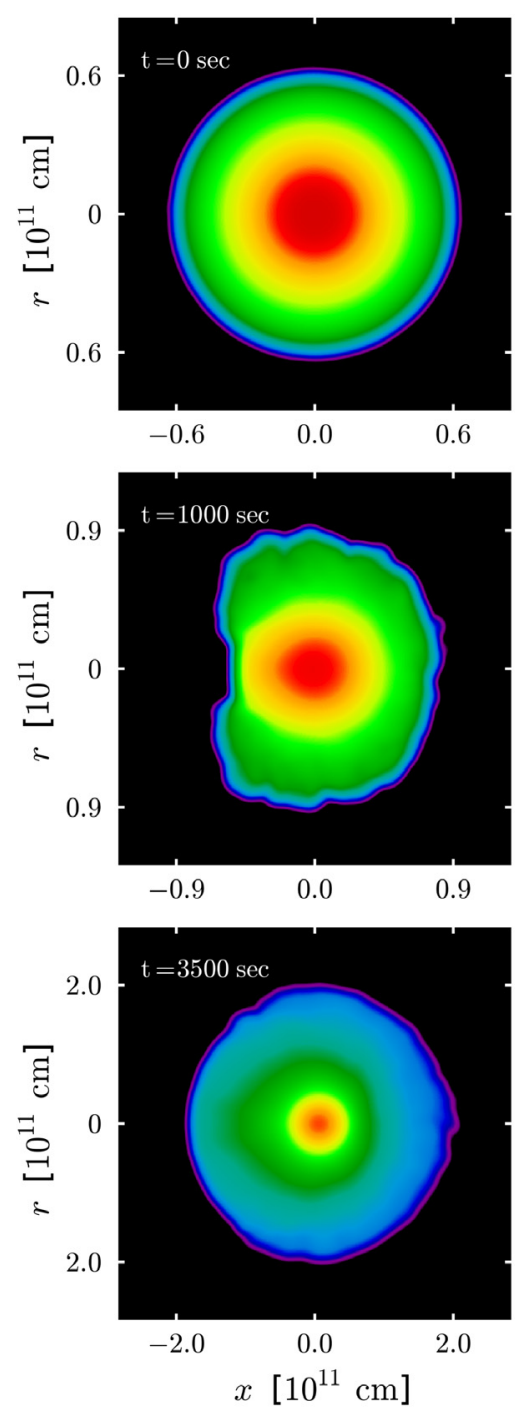
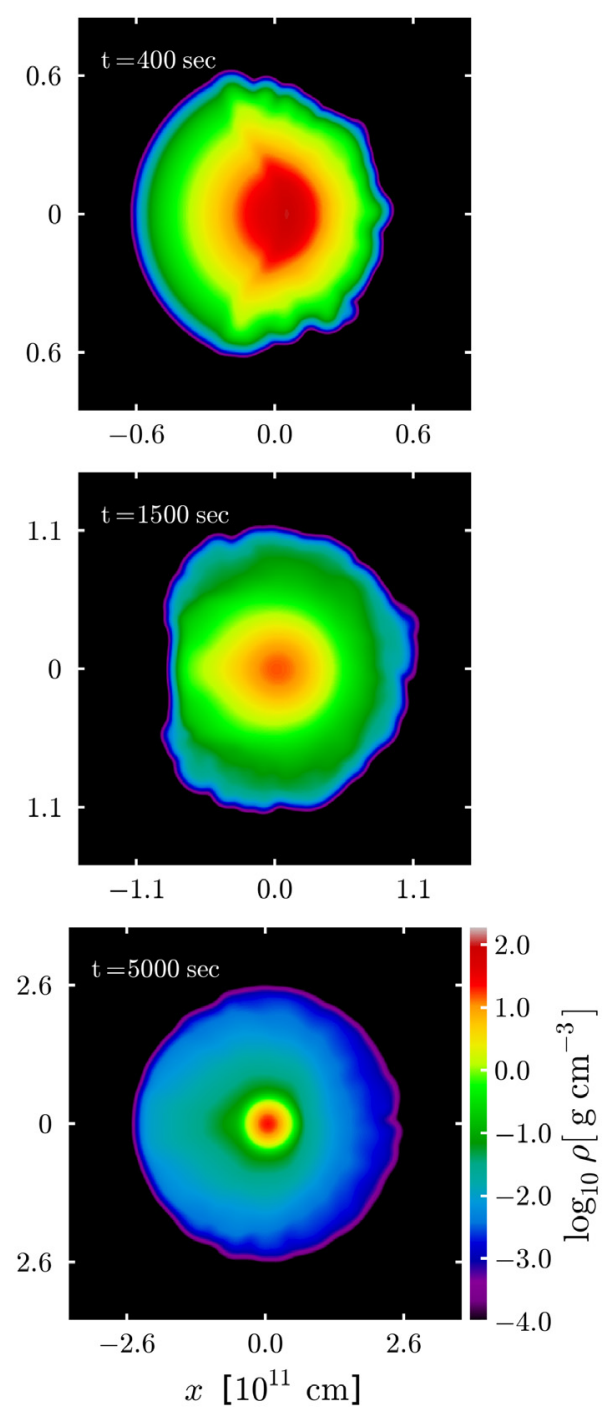

Fig. 13. Density evolution of the remnant star in ms_22a model simulations. Only the bound material that originally belonged to the companion star are included. The logarithm of density is color-coded. model ms_22a. The remnant companion star is puffed up due to the supernova impact and heating, and it has a large radius of $\sim 4 R_{\odot}$ (see the last snapshot of Fig. 13). Although the surviving companion star shrinks and relaxes to be almost spherical again $5000 \mathrm{~s}$ after the explosion, it is out of thermal equilibrium and its density and temperature are asymmetric (see Fig. 13). Podsiadlowski (2003) modeled the post-impact evolution of the surviving companion star and showed that the star is able to completely recover thermal equilibrium $\sim 500 \mathrm{yr}$ after the supernova explosion. He also pointed out that a surviving companion star may be significantly overluminous or underluminous $10^{3}-10^{4} \mathrm{yr}$ after the explosion relative to its pre-supernova luminosity (Podsiadlowski 2003). However, he did not simulate the dynamical interaction of the companion star with the supernova ejecta. In our work, we followed the detailed interaction of SNe Ia and their companion stars. It is necessary to carry out a separate calculation to follow the post-impact evolution of a remnant star during its re-equilibration phase, which will constrain the properties of the surviving companion star for searches in historic SN Ia remnants. This will be addressed in a forthcoming study.

\section{Summary and conclusions}

Based on a range of more realistic companion star models than those used in previous work, we have investigated the impact of
Type Ia supernova ejecta on their companion stars in WD + MS binary systems using the SPH code GADGET3. As an initial model for our hydrodynamical impact simulations, all companion stars are constructed with Eggleton's stellar evolution code incorporating the possibility of an instability of the accretion disk around the WD and including the prescription of Hachisu et al. (1999b) for mass accretion onto the WD. We summarize the basic results and conclusions of our impact simulation as follows.

1. For our binary systems, we always find a stripped mass larger than $0.1 M_{\odot}$. This is in disagreement with the most recent observational constraints on the detection of hydrogen in nebular spectra of SNe Ia (Leonard 2007).

2. Such large stripped masses cause a serious problem for the $\mathrm{SD}$ scenario of SNe Ia. However, prior mass-stripping from the companion star by an optically thick wind (Hachisu et al. 1999a, 2008) or a spin-up spin-down phase as proposed by Justham (2011); Di Stefano et al. (2011); Hachisu et al. (2012) might be able to explain the absence of hydrogen emission in nebular spectra of SNe Ia.

3. For a given companion model, the dependence of stripped mass and kick velocity on the ratio of separation to the radius of the companion, $a_{\mathrm{f}} / R_{2}$, can be fitted by a power law. However, we do not find the same fit parameters as PRHW08 when we put their models and ours together. This indicates 
that details of the structure of companion stars are important for the results of the supernova impact.

4. The differences to previous works are attributed to a more realistic treatment of the binary evolution of our progenitor models.

Further improvements to the binary evolution and observational constraints for more SNe Ia are needed, and more detailed modeling (in particular of the radiative transfer in the nebular phase) is required to determine under which circumstances the hydrogen will be detectable in observations.

Acknowledgements. We thank the referee and the editor for their useful comments which helped to improve the paper. We thank Ken'ichi Nomoto, Achim Weiss and Stefan Taubenberger for useful discussions. Z.W.L. and Z.W.H. thank the financial support from the MPG-CAS Joint Doctoral Promotion Program (DPP) and Max-Planck Institute for Astrophysics (MPA). This work is supported by the National Natural Science Foundation of China (Grant Nos. 2009CB824800, 11033008 and 11103072) and the Chinese Academy of Sciences (Grant No. KJCX2-YW-T24). The work of F.K.R. was supported by Deutsche Forschungsgemeinschaft via the Emmy Noether Program (RO 3676/11) and by the ARCHES prize of the German Federal Ministry of Education and Research (BMBF). The simulations were carried out at the Computing Center of the Max Plank Society, Garching, Germany.

\section{References}

Alexander, D. R., \& Ferguson, J. W. 1994, ApJ, 437, 879

Chen, X.-F., \& Tout, C. A. 2007, Chinese J. Astron. Astrophys., 7, 245

Chomiuk, L., Soderberg, A. M., Moe, M., et al. 2012, ApJ, 750, 164

Chugai, N. N. 1986, SvA, 30, 563

Di Stefano, R., Voss, R., \& Claeys, J. S. W. 2011, ApJ, 738, L1

Eggleton, P. P. 1971, MNRAS, 151, 351

Eggleton, P. P. 1972, MNRAS, 156, 361

Eggleton, P. P. 1973, MNRAS, 163, 279

Finzi, A., \& Wolf, R. A. 1967, ApJ, 150, 115

Geier, S., Nesslinger, S., Heber, U., et al. 2007, A\&A, 464, 299

Geier, S., Heber, U., Kupfer, T., \& Napiwotzki, R. 2010, A\&A, 515, A37

Hachisu, I., Kato, M., \& Nomoto, K. 1996, ApJ, 470, L97

Hachisu, I., Kato, M., \& Nomoto, K. 1999a, ApJ, 522, 487

Hachisu, I., Kato, M., Nomoto, K., \& Umeda, H. 1999b, ApJ, 519, 314

Hachisu, I., Kato, M., \& Nomoto, K. 2008, ApJ, 679, 1390

Hachisu, I., Kato, M., Saio, H., \& Nomoto, K. 2012, ApJ, 744, 69

Han, Z., \& Podsiadlowski, P. 2004, MNRAS, 350, 1301

Hillebrandt, W., \& Niemeyer, J. C. 2000, ARA\&A, 38, 191

Horesh, A., Kulkarni, S. R., Fox, D. B., et al. 2012, ApJ, 746, 21

Hoyle, F., \& Fowler, W. A. 1960, ApJ, 132, 565

Iben, Jr., I., \& Tutukov, A. V. 1984, ApJ, 284, 719

Iglesias, C. A., \& Rogers, F. J. 1996, ApJ, 464, 943

Ivanova, N., \& Taam, R. E. 2004, ApJ, 601, 1058

Justham, S. 2011, ApJ, 730, L34

Kasen, D. 2010, ApJ, 708, 1025

Kato, M. 2011, Proc. IAU Symp. [arXiv: 1110.0055$]$

Kato, M., \& Hachisu, I. 2004, ApJ, 613, L129
Kerzendorf, W. E., Schmidt, B. P., Asplund, M., et al. 2009, ApJ, 701, 1665

Lasota, J.-P. 2001, New ARv, 45, 449

Leibundgut, B. 2008, Gen. Rel. Grav., 40, 221

Leonard, D. C. 2007, ApJ, 670, 1275

Mannucci, F. 2005, in 1604-2004: Supernovae as Cosmological Lighthouses, eds. M. Turatto, S. Benetti, L. Zampieri, \& W. Shea, ASP Conf. Ser., 342, 140

Mannucci, F. 2009, in AIP Conf. Ser. 1111, eds. G. Giobbi, A. Tornambe,

G. Raimondo, M. Limongi, L. A. Antonelli, N. Menci, \& E. Brocato, 467

Maoz, D., \& Badenes, C. 2010, MNRAS, 407, 1314

Maoz, D., \& Mannucci, F. 2012, Publ. Astron. Soc. Austr., 29, 447

Maoz, D., Sharon, K., \& Gal-Yam, A. 2010, ApJ, 722, 1879

Marietta, E., Burrows, A., \& Fryxell, B. 2000, ApJS, 128, 615

Mattila, S., Lundqvist, P., Sollerman, J., et al. 2005, A\&A, 443, 649

Meng, X., \& Yang, W. 2010, ApJ, 710, 1310

Meng, X., Chen, X., \& Han, Z. 2007, PASJ, 59, 835

Mennekens, N., Vanbeveren, D., De Greve, J. P., \& De Donder, E. 2010, A\&A, 515, A 89

Nelemans, G., Napiwotzki, R., Karl, C., et al. 2005, A\&A, 440, 1087

Nomoto, K. 1982, ApJ, 253, 798

Nomoto, K., \& Iben, Jr., I. 1985, ApJ, 297, 531

Nomoto, K., Thielemann, F.-K., \& Yokoi, K. 1984, ApJ, 286, 644

Pakmor, R., Röpke, F. K., Weiss, A., \& Hillebrandt, W. 2008, A\&A, 489, 943

Pakmor, R., Kromer, M., Röpke, F. K., et al. 2010, Nature, 463, 61

Pakmor, R., Hachinger, S., Röpke, F. K., \& Hillebrandt, W. 2011, A\&A, 528, A117

Pakmor, R., Edelmann, P., Röpke, F. K., \& Hillebrandt, W. 2012a, MNRAS, 424, 2222

Pakmor, R., Kromer, M., Taubenberger, S., et al. 2012b, ApJ, 747, L10

Pan, K.-C., Ricker, P. M., \& Taam, R. E. 2010, ApJ, 715, 78

Pan, K.-C., Ricker, P. M., \& Taam, R. E. 2012, ApJ, 750, 151

Patat, F., Chandra, P., Chevalier, R., et al. 2007, Science, 317, 924

Paxton, B., Bildsten, L., Dotter, A., et al. 2011, ApJS, 192, 3

Perlmutter, S., Aldering, G., Goldhaber, G., et al. 1999, ApJ, 517, 565

Phillips, M. M. 1993, ApJ, 413, L105

Phillips, M. M., Lira, P., Suntzeff, N. B., et al. 1999, AJ, 118, 1766

Podsiadlowski, P. 2003 [arXiv:astro-ph/0303660]

Pols, O. R., Tout, C. A., Schroder, K.-P., Eggleton, P. P., \& Manners, J. 1997, MNRAS, 289, 869

Riess, A. G., Filippenko, A. V., Challis, P., et al. 1998, AJ, 116, 1009

Rodríguez-Gil, P., Santander-García, M., Knigge, C., et al. 2010, MNRAS, 407, L21

Ruiter, A. J., Belczynski, K., \& Fryer, C. 2009, ApJ, 699, 2026

Ruiz-Lapuente, P., Comeron, F., Méndez, J., et al. 2004, Nature, 431, 1069

Saio, H., \& Nomoto, K. 1998, ApJ, 500, 388

Schaefer, B. E., \& Pagnotta, A. 2012, Nature, 481, 164

Schroder, K.-P., Pols, O. R., \& Eggleton, P. P. 1997, MNRAS, 285, 696

Springel, V. 2005, MNRAS, 364, 1105

Springel, V., Yoshida, N., \& White, S. D. M. 2001, New Astron., 6, 79

Sternberg, A., Gal-Yam, A., Simon, J. D., et al. 2011, Science, 333, 856

van Paradijs, J. 1996, ApJ, 464, L139

Wang, B., \& Han, Z. 2010, A\&A, 515, A88

Wang, B., Chen, X., Meng, X., \& Han, Z. 2009, ApJ, 701, 1540

Wang, B., Li, X.-D., \& Han, Z.-W. 2010, MNRAS, 401, 2729

Webbink, R. F. 1984, ApJ, 277, 355

Wheeler, J. C., Lecar, M., \& McKee, C. F. 1975, ApJ, 200, 145

Whelan, J., \& Iben, Jr., I. 1973, ApJ, 186, 1007 